\title{
Modified technique for determining therapeutic response to radiofrequency ablation therapy for hepatocellular carcinoma using US-volume system
}

\author{
ATSUSHI HIRAOKA ${ }^{1}$, MASASHI HIROOKA ${ }^{2}$, YOHEI KOIZUMI ${ }^{1}$, SATOSHI HIDAKA ${ }^{1}$, TAKAHIDE UEHARA ${ }^{1}$, \\ SOICHI ICHIKAWA ${ }^{1}$, AKI HASEBE ${ }^{1}$, YASUNAO MIYAMOTO ${ }^{1}$, TOMOYUKI NINOMIYA ${ }^{1}$, \\ KOJIRO MICHITAKA ${ }^{1}$, NORIO HORIIKE ${ }^{2}$, YOSHIMASA YAMASHITA ${ }^{2}$, \\ YOICHI HIASA ${ }^{2}$, BUNZO MATSUURA ${ }^{2}$ and MORIKAZU ONJI ${ }^{2}$ \\ ${ }^{1}$ Department of Gastroenterology, Ehime Prefectural Central Hospital and ${ }^{2}$ Gastroenterology and Metabology, \\ Ehime University Graduate School of Medicine, Ehime, Japan
}

Received March 17, 2009; Accepted June 26, 2009

DOI: 10.3892/or_00000660

\begin{abstract}
In radiofrequency ablation (RFA) for hepatocellular carcinoma (HCC), microbubbles appearing during the procedure make it difficult to determine effectiveness with ultrasonography (US) imaging. We developed a modified US-volume system and evaluated its efficacy for demonstrating response to therapeutic RFA. Our US-volume system displays multiplanar reconstruction (MPR) images providing a synchronized view with a US image along with past USvolume data in real-time side-by-side on a personal computer. Seventy-eight patients (94 nodules) were enrolled, of whom 35 (47 nodules) were evaluated using this system (US-volume group) and compared to the other 43 (47 nodules) examined before development of our system (control group). All nodules were clearly depicted by US. If the shortage of margin was predicted with US-volume system, we performed additional needle insertion. Tumor necrosis following RFA was graded by dynamic computed tomography as follows: Grade A, necrotic area surrounded in all directions with an adequate margin $(\geq 5 \mathrm{~mm})$; Grade $\mathrm{B}$, necrotic area surrounded in all directions, though some margin areas $<5 \mathrm{~mm}$; and Grade $\mathrm{C}$, residual tumor or necrotic area smaller than the target tumor. In the US-volume group, the average tumor size was not smaller than that in the control $(15.9 \pm 4.9$ vs. $16.0 \pm 4.3 \mathrm{~mm})$ and adequate margins were obtained (Grade A, B, C, 45/1/1 vs. $35 / 8 / 4 ; \mathrm{P}<0.01)$. Further, there was a significant reduction in numbers of RFA sessions as compared to the control
\end{abstract}

Correspondence to: Dr Atsushi Hiraoka, Department of Gastroenterology, Ehime Prefectural Central Hospital, Ehime 790-0024, Japan

E-mail: hirage@m.ehime-u.ac.jp

Key words: abdominal imaging, radiofrequency ablation, hepatocellular carcinoma, ultrasonographic volume, multiplanar reconstruction
$(1.03 \pm 0.17$ vs. $1.12 \pm 0.32 ; \mathrm{P}<0.01)$. In $\mathrm{HCC}$ patients undergoing RFA, our modified US-volume system accurately demonstrated therapeutic response, which led to a reduced number of RFA sessions.

\section{Introduction}

Radiofrequency ablation (RFA) is performed for patients with hepatocellular carcinoma (HCC) worldwide as a curative local therapy, because of the low rates of morbidity and mortality, and high level of efficacy (1-4). Local therapeutic response following RFA has been reported as one of the important death-related factors in patients with $\mathrm{HCC}$ (5).

Recently, new therapeutic methods used to assist RFA have been developed that utilize multiplanar reconstruction (MPR) images obtained from volume data provided by multidetector row computed tomography (CT), which is also known as virtual US (VUS) (6). Furthermore, real-time VUS (RVUS) results have been reported $(7,8)$. The development of VUS has made it easier to puncture the invisible tumors and unclear targets in B-mode. On the other hand, the spread of microbubbles during the RFA procedure is a well-known phenomenon that interferes with a clear depiction of the target tumors by US or contrast enhanced US (CEUS). However, in spite of the progress in development of assistant methods, repuncturing in the area of reconstruction during RFA is difficult for the operator due to microbubbles produced by previous ablations. Although US imaging can show puncturing and ablation of tumors, sometimes an adequate margins cannot be obtained, which are determined by dynamic CT following RFA. Recent technological advances such as RVUS have made it possible to display MPR images that provide the same view as US images constructed from past US-volume data in real-time side-by-side with a personal computer. We developed a modified US-volume system (Figs. 1 and 2) to determine therapeutic response and to assist re-puncturing during the RFA procedure in cooperation with Hitachi Medical Corp., Japan, and the Department of Gastrointestinal Oncology, Osaka Medical Center for Cancer and Cardiovascular 


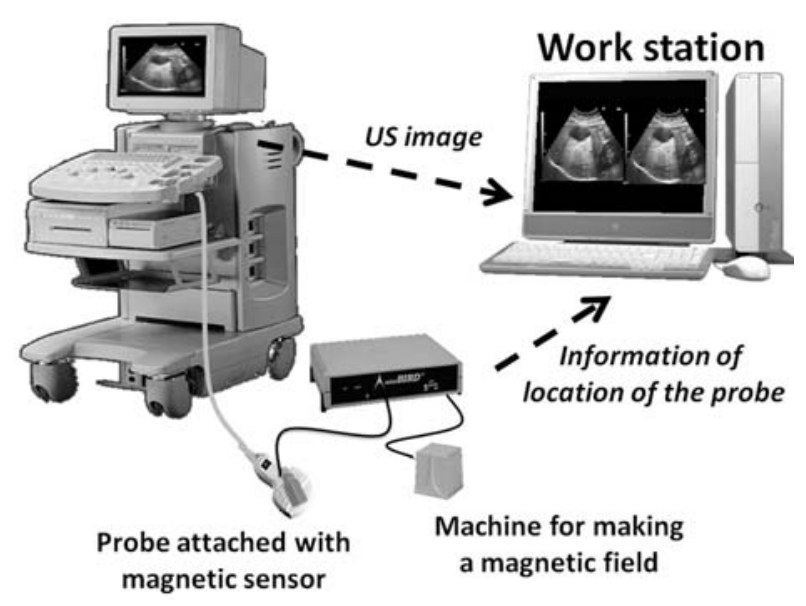

Figure 1. Our US-volume system. An RVUS system with an EUB7500 (Hitachi Medical Corp.) is used to capture US-volume data. The system runs smoothly on a personal computer with Windows XP (Microsoft Co., Ltd.). A synchronized view from US imaging and past US-volume data are shown in real-time side-by-side on a personal computer.

Diseases, Japan (9). In the present study, we evaluated the usefulness for determining and enhancing therapeutic response during RFA procedures using our modified US-volume system.

\section{Materials and methods}

The evaluation protocol with US-volume and data collection was approved by Institutional Review Board (IRB) of Ehime Prefectural Central Hospital, and written informed consent was received from all patients before each procedure.

Patients. Seventy-eight patients with 94 nodules who were evaluated with dynamic CT after RFA from October 2007 to July 2008 were enrolled. All patients were admitted to our hospital, and were selected for RFA based on our strategy (Fig. 3) and following the guidelines for treatment of HCC in Japan (10). The EUB7500 (Hitachi Medical Corp.) was introduced at our hospital in October 2007 and the personal computer used to calculate the present US-volume system was introduced in March 2008. The diagnosis of HCC was based on histology and/or cytological findings, or imaging evidence of tumor formation in the liver (with arterial hyper vascularization) on at least 2 imaging modalities [dynamic CT (11), ultrasonography and angiography]. All nodules in patients enrolled in the present study were clearly revealed by US or CEUS. The patients were divided into the historical control group (control group: $n=43,47$ nodules), who were treated up to March 2008, before introduction of the USvolume system, and US-volume system group (US-volume group: $n=35,47$ nodules), who were treated after introduction of the present system, beginning in March 2008.

US-volume system. To combine past US images with real-time US imaging, we used the RVUS system with an EUB7500 to capture the US-volume data. This system runs smoothly on a personal computer with Windows XP (Microsoft Co., Ltd., Japan). First, US-images of the puncture point or best point where the target lesions were clearly revealed prior to RFA were obtained, and then reconstruction of those images was performed. Then, a synchronized view from US imaging and
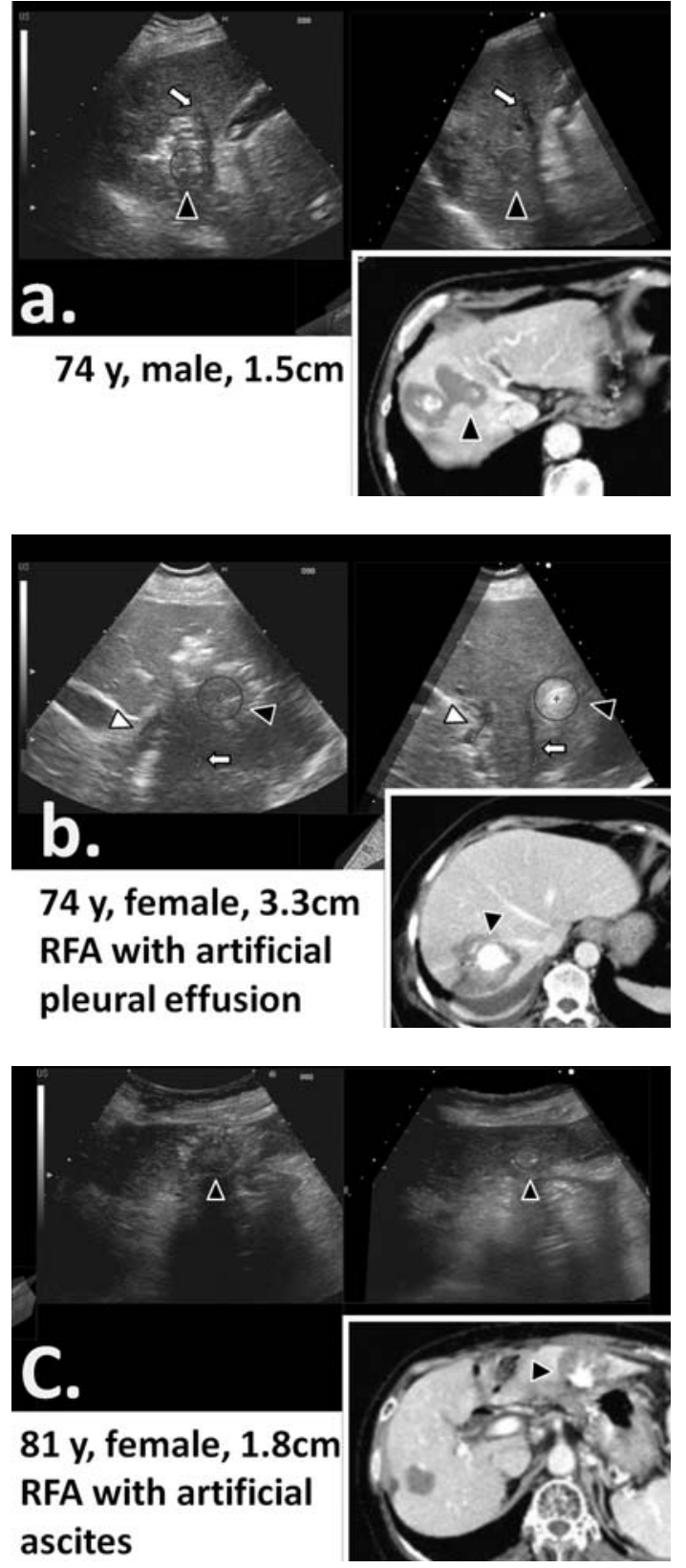

Figure 2. Evaluation of therapeutic response to radiofrequency ablation (RFA). The right side shows past ultrasonography (US)-volume data and the left real-time US data. These are displayed side-by-side in real-time on a personal computer. In the cases of which target lesions were located near portal veins and hepatic veins ( $a$ and $b$ ), we marked line images on portal veins (white arrow head) and hepatic veins (white arrow) on the view of past US-volume data (right side). The marked lines can be automatically displayed on real-time view as an appropriate length depending on the position of probe and make us easy to adjust both views. The sphere (black arrow head) drawn surrounding the target tumor on past US-volume, and also is displayed in both views and microbubbles could be seen surrounding the sphere ( $a, b$ and $c)$. Not only the shape of liver but also the line images were used to adjust between real-time US-images (left side) and past US-volume data (right side). Enough margins were obtained in dynamic computed tomography after RFA procedure.

past US-volume data was produced in real-time side-by-side using a personal computer (Fig. 1). In the present system, we traced a sphere around the center of the target tumor based on US-volume data, so that the maximum diameter of the sphere was the same as the maximum size of the lesion and aligned it parallel to the real-time US image (Fig. 2). In this manner, the marked sphere image can be automatically displayed as 


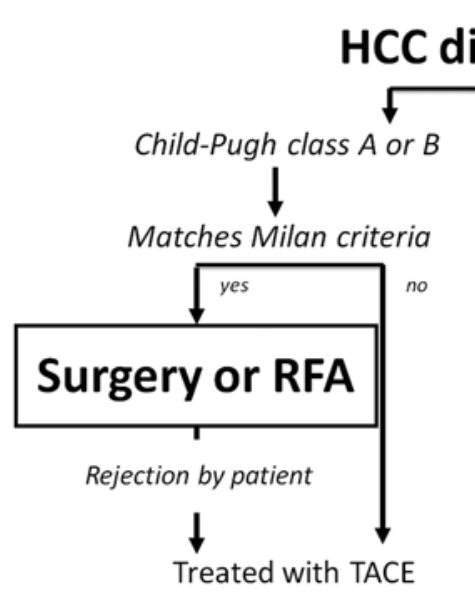

Figure 3. Therapeutic strategy for treatment of HCC based on therapeutic guidelines used in Japan. RFA, radiofrequency ablation; TACE, transcatheter arterial chemoembolization; TAI, transcatheter arterial chemo infusion.

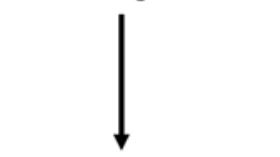

Liver transplantation TAI

Best supportive therapy

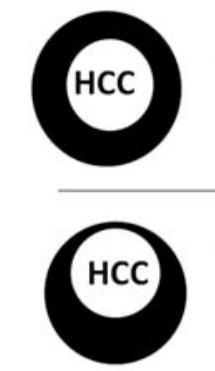

\section{Grade A}

Target tumor was surrounded by necrotic area in all directions with enough margin ( $\geq 5 \mathrm{~mm}$ ).

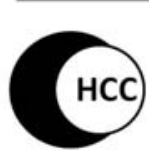

\section{Grade C}

Necrotic area was smaller than target tumor, or ablation was incomplete.

Figure 4. Determination of tumor necrosis. We determined the amount of tumor necrosis following RFA according to 3 grades of response to RFA using dynamic computed tomography.

after ablation. If the shortage of margin was predicted by USvolume system, we performed additional needle insertion to the different site of the target until the margin was judged adequate in US-volume. line images on the vessels in view of past US-volume data and adjusted between real-time images and past US-volume data using with not only the shape of liver but also the line images. The marked lines can be automatically displayed on real-time view as an appropriate length depending on the position of probe and make us easy to adjust both views. We always attach and use a probe at the same position and angle of the body of the patient to evaluate the results of RFA.

$R F A$. Prior to RFA treatment, $15 \mathrm{mg}$ of pentazocine hydrochloride and $25 \mathrm{mg}$ of hydroxyzine hydrochloride were administrated intramuscularly. Local anesthesia was induced by $5 \mathrm{ml}$ of $1 \%$ lidocaine injected through the skin into the peritoneum along a predetermined puncture line. In cases in which the tumor was difficult to visualize using conventional US, we used CEUS (12) with Sonazoid ${ }^{\circledR}(13,14)$ and/or artificial pleural effusion (15) and ascites as assistant methods for RFA before capturing the US-volume data. Midazolam (Dormicum ${ }^{\circledR}$, Astellas Pharma Inc., Japan) was injected intravenously at the start of ablation $(0.1 \mathrm{mg} / \mathrm{kg})$. Most of hypervascular nodules were subjected to transcatheter arterial chemoembolization (TACE) $(16,17)$ with Epirubicin-Lipiodol emulsion and multiporous gelatine particles $\left(\right.$ Gelpart $^{\circledR}$, Astellas Pharma Inc., Japan) before RFA. RFA was performed with sonographic guidance (EUB7500) using a combex or microcombex probe. We inserted a 20 -cm long 17 -gauge radiofrequency electrode equipped with a 2- or 3-cm long exposed metallic tip (Radionics Cool-tip, Burlington, MA, USA). In control group, we finished the RFA procedure when the operator judged microbubbles were spread enough around the target lesions in real-time images. In US-volume group, after adjusting the images between real-time US and USvolume images based on the shape of the liver, surrounding organs, or intrahepatic vessels, evaluation of response to RFA was performed and judged adequate if the maximum diameter of the traced sphere on the US-volume image, which matched the maximum size of the target tumor, was surrounded adequately by hyperechoic microbubbles $5 \mathrm{~min}$
Estimation of therapeutic effect. Dynamic CT was performed for all nodules at 3-5 days after RFA. The necrotic area of the liver parenchyma surrounding the target lesion was revealed as hypoattenuating during the portal phase of dynamic CT. When a necrotic area larger than the viable area was obtained on post-treatment dynamic CT as compared to pre-treatment dynamic CT, the therapy was considered to be successful (18). Two expert radiologists who specialize in CT imaging checked the post-treatment dynamic CT results. Furthermore, we determined tumor necrosis after RFA according to three grades of response for treatment, i) the necrotic area was surrounded in all directions, excluding the parts which were next to hepatic vein and/or Grisson's sheath or surface of liver, with a margin $\geq 5 \mathrm{~mm}$ (Grade $\mathrm{A}$ ), ii) the necrotic area was surrounded in all directions, excluding the parts which were next to hepatic vein and/or Grisson's sheath or surface of liver, though some margin areas were $<5 \mathrm{~mm}$ (Grade B), and iii) the residual tumor or necrotic area was smaller than the target tumor (Grade C) (Fig. 4). Additional RFA was performed when the results of dynamic CT were judged as Grade C, or as Grade B with a very thin margin with the appearance of a line. Informed consent for the RFA therapy was obtained from all patients.

Statistical analysis. The data are expressed as the mean \pm SD. Statistical analyses were performed using Student's t-test for unpaired data and a Mann-Whitney U test as appropriate. All statistical analyses were performed by SPSS 16.0J (SPSS Japan Inc., Japan). A P-value of $<0.05$ was considered to represent statistical significance.

\section{Results}

Following introduction of our US-volume system, 47 nodules, which were revealed clearly using US with or without assistant methods (e.g. CEUS, artificial pleural effusion), were ablated 
Table I. Laboratory data for patients undergoing RFA procedures.

\begin{tabular}{|c|c|c|c|}
\hline & $\begin{array}{l}\text { US-volume group } \\
(\mathrm{n}=35,47 \text { nodules })\end{array}$ & $\begin{array}{l}\text { Control group } \\
(\mathrm{n}=43,47 \text { nodules })\end{array}$ & P-value \\
\hline Age (years) & $69.0 \pm 9.5$ & $69.1 \pm 10.2$ & 0.442 \\
\hline Gender (male/female) & $26 / 9$ & $26 / 17$ & 0.282 \\
\hline Positive for anti-HCVAb & $30 / 5$ & $37 / 6$ & 0.967 \\
\hline T-bil (mg/dl) & $0.89 \pm 0.52$ & $0.86 \pm 0.45$ & 0.586 \\
\hline AST (IU/l) & $44.1 \pm 28.3$ & $51.1 \pm 32.1$ & 0.041 \\
\hline ALT (IU/l) & $54.0 \pm 30.0$ & $60.4 \pm 35.8$ & 0.442 \\
\hline $\mathrm{Alb}(\mathrm{g} / \mathrm{dl})$ & $3.78 \pm 0.47$ & $3.82 \pm 0.41$ & 0.339 \\
\hline PT $(\%)$ & $75.2 \pm 10.2$ & $74.1 \pm 10.1$ & 0.923 \\
\hline Plt $\left(\mathrm{x} 10^{4} / \mu 1\right)$ & $12.6 \pm 7.5$ & $11.4 \pm 4.9$ & 0.121 \\
\hline AFP (ng/ml) & $103.5 \pm 280.2$ & $57.9 \pm 104.4$ & 0.087 \\
\hline Child Pugh classification (A/B) & $26 / 9$ & $33 / 10$ & 0.937 \\
\hline No. of RFA sessions with contrast enhanced US & 19 & 8 & 0.022 \\
\hline $\begin{array}{l}\text { No. of RFA sessions with artificial pleural } \\
\text { effusion or ascites }\end{array}$ & 18 & 16 & 0.830 \\
\hline No. of nodules treated with TACE before RFA & 34 & 35 & 0.816 \\
\hline Diameter of HCC (mm) & $\begin{array}{c}15.9 \pm 4.9 \\
\text { (range, } 10-28)\end{array}$ & $\begin{array}{c}16.0 \pm 4.3 \\
\text { (range, } 9-24)\end{array}$ & 0.421 \\
\hline Segment of tumor (S1-S4/S5, S8/S6, S7) & $14 / 17 / 16$ & $10 / 17 / 20$ & 0.302 \\
\hline No. of RFA sessions & $1.03 \pm 0.17$ & $1.12 \pm 0.32$ & 0.003 \\
\hline
\end{tabular}

AST, aspartate aminotransferase; ALT, alanine aminotransferase; Alb, albumin; PT, prothrombin time; Plt, platelet count; AFP, $\alpha$-fetoprotein; anti-HCVAb, anti-hepatitis $\mathrm{C}$ virus antibody; RFA, radiofrequency ablation; TACE, transcatheter arterial chemoembolization.

Table II. Results of RFA therapy.

\begin{tabular}{lc}
\hline US-volume group (47 nodules) & $45 / 1 / 1$ \\
Grade A/B/C & \\
Control group (47 nodules) & $35 / 8 / 4$ \\
Grade A/B/C &
\end{tabular}

The P-value was under 0.01 in comparisons between the groups.

and evaluated with the present system. No differences were found for age, positive rate for anti-HCV (hepatitis $\mathrm{C}$ virus), hepatic reserve function test, the frequency of TACE before RFA, Child Pugh classification, $\alpha$-fetoprotein (AFP), segment of tumor, or diameter of HCC nodules between the groups (Table I). While the number of CEUS were larger in USvolume group than control $(\mathrm{P}<0.05)$, all nodules were clearly revealed in the RFA procedure.

After determining that the effect of RFA was adequate during the procedure, 2 nodules from the US-volume group and 12 from the control group were found to have an inadequate margin. In the US-volume group, 45 patients had a response grade of $\mathrm{A}$, while 1 was $\mathrm{B}$ and 1 was $\mathrm{C}$, whereas 35 patients in the control group had a response grade of $\mathrm{A}$, while 8 were $\mathrm{B}$ and 4 were $\mathrm{C}(\mathrm{P}<0.01)$ (Table II). Furthermore, the ablated margin obtained around the tumor following RFA was adequate in the US-volume group. Two cases in the
US-volume group did not have an adequate margin. In this time, 1 nodule of control group showed local recurrence, whereas none in US-volume group. The average number of RFA sessions was decreased from $1.12 \pm 0.32$ (control group) to $1.03 \pm 0.17$ (US-volume group) $(\mathrm{P}<0.01)$ after introduction of the present system.

\section{Discussion}

Recent progress in the development of diagnostic and therapeutic modalities for HCC has been remarkable, and RFA is now used worldwide because of its lower invasiveness and equal efficacy to surgical resection (19-21). CEUS with Sonazoid ${ }^{\circledR}$ makes it possible to diagnose small HCCs $(<2 \mathrm{~cm})$ and make continuous images for target lesions in Kupffer phase (14), and is useful as an indicator and guide for RFA (12). Furthermore, artificial pleural effusion and/or artificial ascites have been shown to be helpful for performing percutaneous RFA (15), while guidance provided by VUS (6) and RVUS (7) have been reported as useful for increasing the reliability of RFA in cases with invisible tumors. Although the use of VUS increases the ability for detecting the invisible tumors, development of an effective method to determine therapeutic response for detectable target lesions during the RFA procedure is necessary, which would make it possible to obtain an adequate ablated margin and thus reduce the number of more additional RFA sessions and increase the safety of the re-puncturing. 
Though, CEUS reveals a clear view of target nodules, microbubble appearance during ablation hampers the ability to clearly visualize the entire body of the target lesion during the RFA procedure in CEUS as well as RFA in B-mode of US. The spread of the hyperechoic area by ablation was reported to be useful to estimate the boundaries of the ablated area $(22,23)$, while Nouso et al reported that the size of the area of hyper-echogenicity at $5 \mathrm{~min}$ after ablation and that of the defect observed by CEUS 3-5 days after RFA were closely correlated (24). These findings led us to use hyper-echoic microbubbles at $5 \mathrm{~min}$ after ablation for determining the area of necrosis.

We developed the present US-volume system that synchronizes previously captured US images just before the initial puncture with real-time US imaging for assistance during RFA procedures, and this is the first report for determining the therapeutic response using with modified USvolume system. During the procedure, we place a spherical mark to surround the target tumor that matches the maximum size, and then display the image in parallel with real-time US data using a personal computer. We consider that the margin is adequate if the drawn spherical image is surrounded by a hyperechoic area 5 min after RFA, then finish the procedure. Indeed, the present results in comparison with those of a historical control group showed that a safety margin was more surely obtained and the number of additional RFA sessions reduced. Although there was a weak point in the present system that the target nodules are limited in detectable tumors in US or CEUS without cooperative breath with patient efforts. In the cases difficult to determine the edge of tumors, we used CEUS with Sonazoid before RFA in both groups. Although the number of nodules, which were difficult to reveal clearly in B-mode, were larger in the US-volume group, the US-volume group had better results than the control. The reason for this result was thought to be that additional insertion was performed adequately in the same session based on US-volume data. As a result, we believe that the therapeutic response to RFA was enhanced and cost decreased, because of a shorter hospital stay and reduced number of dynamic CT procedures.

Two cases in the US-volume group did not have an adequate margin, of which 1 (Grade C) was located on top of the hepatic dome near the beating heart and the other (Grade B) was on the surface of the liver, which could only be detected when the patient held their breath. Thus, there are some weak points with the present system in such cases, however, it is considered to be a useful tool for determining and enhancing the efficacy of percutaneous RFA.

In conclusion, our novel US-volume system was found able to determine and enhance therapeutic response to RFA performed for HCC nodules and its utilization is expected to reduce the number of additional RFA sessions.

\section{References}

1. Rossi S, Di Stani M, Buscarni E, et al: Percutaneous RF interstitial thermal ablation in the treatment of hepatic cancer. AJR 167: 759-776, 1996.

2. Tateishi R, Shiina $\mathrm{S}$, Teratani $\mathrm{T}$, et al: Percutaneous radiofrequency ablation for hepatocellular carcinoma. An analysis of 1000 cases. Cancer 103: 1201-1209, 2005.
3. Shiina S, Teratani T, Obi S, Hamamura K, Koike Y and Omata M: Non-surgical treatment of hepatocellular carcinoma: from percutaneous ethanol injection therapy and percutaneous microwave coagulation therapy to radiofrequency ablation. Oncology 62: 64-68, 2002.

4. Ng KK and Poon RT: Role of radiofrequency ablation for liver malignancies. Surg Practice 9: 94-103, 2005.

5. Takahashi S, Kudo M, Chung H, et al: Initial treatment response is essential to improve survival in patients with hepatocellular carcinoma who underwent curative radiofrequency ablation therapy. Occology 72: 98-103, 2007.

6. Hirooka M, Iuchi H, Kumagi T, et al: Virtual sonographic radiofrequency ablation of hepatocellular carcinoma visualized on CT but not on conventional sonography. AJR 186: S255-S260, 2006.

7. Iwasaki T, Mikami E, Shimosegawa T, Arai O and Mitake T: Real-time virtual sonography: a novel navigation tool in percutaneous radiofrequency ablation of hepatocellular carcinomas. Radiological Society of North America Scientific Assembly and Annual Meeting Program. OakBrook, Ill: Radiological Society of North America, p392, 2004.

8. Kitada T, Murakami T, Kuzushita N, et al: Effectiveness of real-time virtual sonography-guided radiofrequency ablation treatment for patients with hepatocellular carcinomas. Hepatol Res 38: 565-571, 2008.

9. Imanaka K, Kasugai H, Tamai C, Matsunaga T and Iishi H: New diagnostic system of evaluation on radiofrequency ablation of hepatocellular carcinoma. Hepatology International 1: 59, 2007.

10. Makuuchi M and Kokudo N: Clinical practice guidelines for hepatocelluar carcinoma: the first evidence based guidelines from Japan. World J Gastroenterol 12: 828-829, 2006.

11. Bruix $\mathbf{J}$ and Sherman M: Management of hepatocellular carcinoma. Hepatology 42: 1208-1236, 2005.

12. Minami Y, Kudo M, Kawasaki T, et al: Percutaneous radiofrequency ablation guided by contrast-enhanced harmonic sonography with artificial pleural effusion for hepatocellular carcinoma in the hepatic dome. AJR 182: 1224-1226, 2004.

13. Numata K, Morimoto M, Ogura T, et al: Ablation therapy guided by contrast-enhanced sonography with Sonazoid for hepatocellular carcinoma lesions not detected by conventional sonography. J Ultrasound Med 27: 395-406, 2008.

14. Kan M, Horiike N, Hiraoka A, et al: Comparison diagnostic efficacy of contrast enhances ultrasonography with Perflubutane and dynamic computed tomography in patients with liver tumors smaller than $2 \mathrm{~cm}$. Hepatology Int 2: A255, 2008.

15. Uehara T, Hirooka M, Ishida K, et al: Percutaneous ultrasoundguided radiofrequency ablation of hepatocellular carcinoma with artificially induced pleural effusion and ascites. J Gastroenterol 42: 306-311, 2007.

16. Takayasu K, Arii S, Ikai I, et al: Prospective cohort study of transarterial chemoembolization for unresectable hepatocellular carcinoma in 8510 patients. Gastroenterology 131: 461-469, 2006.

17. Hiraoka A, Kumagi T, Hirooka M, et al: Prognosis following transcatheter arterial embolization for 121 patients with unresectable hepatocellular carcinoma with or without a history of treatment. World J Gastroenterol 12: 2075-2079, 2006.

18. Ebara M, Kita K, Sugiura N, et al: Therapeutic effect of percutaneous ethanol injection on small hepatocellular carcinoma: evaluation with CT. Radiology 195: 371-377, 1995.

19. Hiraoka A, Horiike N, Yamashita Y, et al: Efficacy of radiofrequency ablation therapy compared to surgical resection in 164 patients in Japan with single hepatocellular carcinoma smaller than $3 \mathrm{~cm}$, along with report of complications. HepatoGastroenterol 55: 2171-2174, 2008.

20. Chen MS, Li QZ, Zheng Y, et al: A prospective randomized trial comparing percutaneous local ablative therapy and partial hepatectomy for small hepatocellular carcinoma. Ann Surg 243: 321-328, 2006.

21. Livraghi T, Meloni F, Stasi MD, et al: Sustained complete and complications rates after radiofrequency ablation of very early hepatocellular carcinoma in cirrhosis: is resection still the treatment of choice? Hepatology 47: 82-89, 2008.

22. Mcghana JP and Dodd GD: Radiofrequency ablation of the liver: current status. AJR 176: 3-16, 2001.

23. Livraghi T, Goldberg SN, Lazzaroni S, et al: Small hepatocellular carcinoma: treatment with radio-frequency ablation versus ethanol injection. Radiology 210: 655-661, 1999.

24. Nouso K, Shiraga K, Uematsu S, et al: Prediction of the ablated area by the spread of microbubbles during radiofrequency ablation of hepatocellular carcinoma. Liver Int 25: 967-972, 2005. 